Cahiers $d u$ MONDE RUSSE

\section{Cahiers du monde russe}

Russie - Empire russe - Union soviétique et États indépendants

$53 / 4 \mid 2012$

Varia

\title{
Robert O. Crummey, Old Believers in a Changing World
}

Aleksandr Lavrov

\section{OpenEdition}

\section{Journals}

Édition électronique

URL : http://journals.openedition.org/monderusse/7736

DOI : $10.4000 /$ monderusse. 7736

ISSN : $1777-5388$

Éditeur

Éditions de l'EHESS

\section{Édition imprimée}

Date de publication : 15 décembre 2012

ISSN : $1252-6576$

Référence électronique

Aleksandr Lavrov, «Robert O. Crummey, Old Believers in a Changing World», Cahiers du monde russe [Онлайн], 53/4 | 2012, Выложить онлайн 19 mars 2013, Наводить справки в 22 septembre 2020. URL : http://journals.openedition.org/monderusse/7736 ; DOI : https://doi.org/10.4000/monderusse. 7736

Ce document a été généré automatiquement le 22 septembre 2020.

(c) École des hautes études en sciences sociales 


\title{
Robert O. Crummey, Old Believers in a Changing World
}

\author{
Aleksandr Lavrov
}

\section{RÉFÉRENCE}

Robert O. CRUMmeY, Old Believers in a Changing World. DeKalb : Northern Illinois University Press, 2011, 267 p.

1 ПЕРЕД НАМИ ЧЕТВЕРТАЯ КНИГА РОБЕРТА КРАММИ. КАЖДАЯ ИЗ ПРЕДЫДУЩИХ КНИГ АВТОРА (ИССЛЕДОВАНИЕ О ВЫГОВСКОЙ СТАРООБРЯДЧЕСКОЙ ПУСТЫНИ, МОНОГРАФИЯ О МОСКОВСКОМ БОЯРСТВЕ В 1613-1689 ГГ. И УЧЕБНИК ПО РУССКОЙ ИСТОРИИ, ОХВАТЫВАЮЩИЙ ВРЕМЯ ВОЗВЫШЕНИЯ МОСКВЫ${ }^{1}$ ) СТАЛА В СВОЕ ВРЕМЯ ИСТОРИОГРАФИЧЕСКИМ СОБЫТИЕМ И СУЩЕСТВЕННО ИЗМЕНИЛА ОБСТАНОВКУ В ТОЙ УЗКОЙ ОБЛАСТИ, КОТОРОЙ ОНА БЫЛА ПОСВЯЩЕНА. ТЕМ ВЫШЕ УРОВЕНЬ ОЖИДАНИЙ, СВЯЗАННЫХ С НОВОЙ ПУБЛИКАЦИЕЙ.

2 РЕЦЕНЗИРУЕМАЯ КНИГА ВКЛЮЧАЕТ В СЕБЯ СТАТЬИ 1980-2000-Х ГОДОВ (НОВОЙ ЯВЛЯЕТСЯ ГЛАВА «НОВОСИБИРСКАЯ ШКОЛА ИССЛЕДОВАНИЙ СТАРООБРЯДЧЕСТВА», С. 187-189), И ПРЕДСТАВЛЯЕТ СВОЕГО РОДА ВОЗВРАТ К НАЧАЛУ - К ВОПРОСУ О РОЛИ СТАРООБРЯДЧЕСТВА В ИСТОРИИ РОССИИ В НОВОЕ ВРЕМЯ. ТОТ ФАКТ, ЧТО БОЛЬШАЯ ЧАСТЬ РАБОТ, ВКЛЮЧЕННЫХ В КНИГУ, УЖЕ БЫЛА ОПУБЛИКОВАНА В ЖУРНАЛАХ ИЛИ СБОРНИКАХ СТАТЕЙ, СТАВИТ НЕМАЛОВАЖНЫЙ ВОПРОС О СТРУКТУРЕ. АВТОР ПРЕДПОЧЕЛ ТЕМАТИЧЕСКУЮ СТРУКТУРУ, ПОМЕСТИВ СТАТЬИ О РАННЕМ СТАРООБРЯДЧЕСТВЕ В НАЧАЛЕ КНИГИ. МНЕ БОЛЕЕ ПО ВКУСУ ПРИШЕЛСЯ БЫ ПРИНЦИП «СОБРАНИЯ СОЧИНЕНИЙ», ПРИ КОТОРОМ РАБОТЫ БЫЛИ БЫ ПРЕДСТАВЛЕНЫ В ПОРЯДКЕ ИХ ОПУБЛИКОВАНИЯ АВТОРОМ. БОЛЕЕ ТОГО, КАК ЧИТАТЕЛЬ Я ПРЕДПОЧИТАЮ ИЗДАТЕЛЬСКОЕ РЕШЕНИЕ, ИЗБРАННОЕ ИЗДАТЕЛЯМИ ЛОНДОНСКОЙ СЕРИИ Variorum reprints, КОТОРЫЕ ДАЖЕ СТАТЬИ ИЗ ЖУРНАЛОВ ПЕРЕИЗДАЮТ В ИХ И СХОДНОМ ТИПОГРАФСКОМ ВИДЕ. МОЖНО СКАЗАТЬ, ЧТО ЭТА ПРОБЛЕМА РЕШЕНА БЛАГОДАРЯ СПИСКУ ПЕРЕИЗДАННЫХ РАБОТ, В КОТОРОМ АВТОР УКАЗАЛ НЕ ТОЛЬКО 
БИБЛИОГРАФИЧЕСКИЕ ДАННЫЕ ПЕРВЫХ ПУБЛИКАЦИЙ, НО И ПРОСТАВИЛ ДАТЫ НАПИСАНИЯ КАЖДОГО ТЕКСТА. ТЕМ НЕ МЕНЕЕ, ИМЕННО ИНОЙ ПОРЯДОК РАСПОЛОЖЕНИЯ СТАТЕЙ ДАЛ БЫ ВОЗМОЖНОСТЬ ВЫПУКЛЕЕ ПРЕДСТАВИТЬ ДВЕ ПРОБЛЕМЫ - РАЗВИТИЕ ВЗГЛЯДОВ САМОГО АВТОРА И ЕГО ВЛИЯНИЕ НА ИСТОРИОГРАФИЧЕСКИЙ КОНТЕКСТ. ВСЕ ЭТО НЕ ЯВЛЯЕТСЯ БУКВОЕДСТВОМ, ПОСКОЛЬКУ САМ АВТОР ХОРОШО ПОНИМАЕТ МЕСТО СВОИХ РАБОТ, ЧТО И ДОКАЗЫВАЕТ, ПОЛНОСТЬЮ ОТКАЗАВШИСЬ ОТ КАКИХ-ЛИБО ВСТАВОК В ТЕКСТЫ СТАТЕЙ. ЕМУ ЧУЖДО ИСКУШЕНИЕ УЧЕСТЬ post factum ЗАМЕЧАНИЯ ОППОНЕНТОВ И ПРЕДСТАВИТЬ СЕБЯ ДАЛЬНОВИДНЕЕ. ИМЕННО ТАК И ПРОИЗОШЛО СО СТАТЬЯМИ О СТАРООБРЯДЧЕСТВЕ КАК «НАРОДНОЙ РЕЛИГИИ» И «ТЕКСТУАЛЬНОМ СООБЩЕСТВЕ», КОТОРЫЕ ПОСЛУЖИЛИ ОТПРАВНОЙ ТОЧКОЙ ДЛЯ КРИТИКИ ГЕОРГА МИХЕЛЬСА, СФОРМИРОВАВШЕГО ИЗ СВОЕГО ОТРИЦАНИЯ ОБОИХ ЭТИХ ПОЛОЖЕНИЙ ОСНОВУ СОБСТВЕННОЙ КОНЦЕПЦИИ РАННЕГО СТАРООБРЯДЧЕСТВА. ВО ВСЕХ ЭТИХ СЛУЧАЯХ КРАММИ ВОСПРОИЗВОДИТ СВОЙ ИСХОДНЫЙ ТЕКСТ БЕЗ ИЗМЕНЕНИЙ - ПОДХОД, КОТОРЫЙ НАВЕРНЯКА ПРИВЕТСТВОВАЛИ БЫ ЕГО ГЕРОИ, СТАРООБРЯДЦЫ.

ПЕРВЫЙ БЛОК СБОРНИКА, ПОСВЯЩЕННЫЙ ИСТОРИОГРАФИИ И ТЕОРЕТИЧЕСКИМ МОДЕЛЯМ СТАРООБРЯДЧЕСТВА, ВКЛЮЧАЕТ ДВЕ СТАТЬИ. В ПЕРВОЙ ИЗ НИХ МОЖНО НАЙТИ МНОГО ИНТЕРЕСНЫХ ЗАМЕТОК - НАПРИМЕР, О РАБОТАХ С. ЗЕНЬКОВСКОГО, КОТОРЫЕ АВТОР СЧИТАЕТ НЕ НАШЕДШИМИ СВОЕГО ЧИТАТЕЛЯ, ПОСКОЛЬКУ ОНИ НЕ ДОХОДИЛИ РУССКОЙ АУДИТОРИИ, НАВЕРНЯКА ОЦЕНИВШЕЙ БЫ ЕГО «НЕОСЛАВЯНОФИЛЬСКИЙ ПОДХОД». ПО МНЕНИЮ КРАММИ, В РАБОТАХ М. ЧЕРНЯВСКИ И ЕГО СОВРЕМЕННИКОВ ПРОИСХОДИТ ОТКАЗ ОТ МОНИСТИЧЕСКИХ, ОДНОФАКТОРНЫХ КОНЦЕПЦИЙ ПРОИСХОЖДЕНИЯ СТАРООБРЯДЧЕСТВА, СВОЙСТВЕННЫХ РОССИЙСКОЙ ИСТОРИОГРАФИИ В ХІХВ. МОЖНО ТОЛЬКО СОГЛАСИТЬСЯ С ОСНОВНЫМ ВЫВОДОМ АВТОРА О ТОМ, ЧТО СОВРЕМЕННАЯ ИСТОРИОГРАФИЯ СТАРООБРЯДЧЕСТВА СОВЕРШЕННО ИНТЕРНАЦИОНАЛЬНА И ПРЕДСТАВЛЯЕТ СОБОЙ ДИНАМИЧНО РАЗВИВАЮЩУЮСЯ ОБЛАСТЬ ИСТОРИЧЕСКОГО ЗНАНИЯ И ЧТО ОСНОВНОЙ ПРОБЛЕМОЙ ЗДЕСЬ ОСТАЕТСЯ НЕДОСТАТОК ФИНАНСИРОВАНИЯ (С.6). ПРИ ЭТОМ НЕЛЬЗЯ НЕ СОЖАЛЕТЬ О ТОМ, ЧТО КРАММИ ОПУСКАЕТ НАИБОЛЕЕ «ЕРЕТИЧЕСКИЕ» ИССЛЕДОВАНИЯ - В ТОМ ЧИСЛЕ СПОРНУЮ И НЕРОВНУЮ РАБОТУ БЕЗВРЕМЕННО УШЕДШЕЙ ИЗ ЖИЗНИ ГАБРИЭЛЕ ШАЙДЕГГЕР (1952-2007), КОТОРАЯ ОКАЗАЛАСЬ ВООБЩЕ ЗАМОЛЧАННОЙ В ИСТОРИОГРАФИИ ПОСЛЕДНИХ ЛЕТ ${ }^{2}$. СРАЗУ ЖЕ ВСЛЕД ЗА НЕЙ СЛЕДУЕТ ОПУБЛИКОВАННАЯ В 1993 Г. И СТАВШАЯ ХРЕСТОМАТИЙНОЙ СТАТЬЯ «СТАРООБРЯДЧЕСТВО КАК НАРОДНАЯ РЕЛИГИЯ: НОВЫЕ ПОДХОДЫ», НАПИСАННАЯ НА ВОЛНЕ ИНТЕРЕСА К «НАРОДНОЙ РЕЛИГИИ», СВЯЗАННОГО С РАБОТАМИ РОБЕРА МЮШАМБЛЕ, КАРЛО ГИНЗБУРГА И А.Я. ГУРЕВИЧА. ПОДХОД КРАММИ БЛИЖЕ НЕ К МЮШАМБЛЕ С ЕГО ПРОТИВОПОСТАВЛЕНИЕМ «НАРОДНОЙ» И «ЭЛИТАРНОЙ» КУЛЬТУР, А К ГИНЗБУРГУ, ПОКАЗЫВАЮЩЕМУ ИХ СЛОЖНОЕ ВЗАИМОДЕЙСТВИЕ. ПОНИМАЯ ИННОВАЦИОННЫЙ ХАРАКТЕР СВОЕГО ТЕЗИСА, КРАММИ САМ ФОРМУЛИРУЕТ КОНТРДОВОДЫ. ВО-ПЕРВЫХ, ДОКУМЕНТАЦИЯ О РАННЕМ СТАРООБРЯДЧЕСТВЕ ОБРЫВОЧНА, ВО-ВТОРЫХ, В НАЧАЛЕ СВОЕЙ ИСТОРИИ ОНО ЗАЧАСТУЮ ФОРМИРОВАЛОСЬ ВЫХОДЦАМИ ИЗ КЛИРА, В-ТРЕТЬИХ, САМО СТАРООБРЯДЧЕСТВО С САМОГО НАЧАЛА НАСТОЛЬКО ГЕТЕРОГЕННО (С. 20), ЧТО УСКОЛЬЗАЕТ ОТ ОБЩИХ ОПРЕДЕЛЕНИЙ. В ТОЙ ЖЕ СТАТЬЕ КРАММИ ПРЕДЛАГАЕТ И ДРУГОЕ, АЛЬТЕРНАТИВНОЕ ЧТЕНИЕ СТАРООБРЯДЧЕСТВА КАК «ТЕКСТУАЛЬНОГО СООБЩЕСТВА» (textual community), ЗАИМСТВОВАННОЕ ИЗ РАБОТ БРАЙАНА СТОКА (Brian Stock). ТЕМ САМЫМ КРАММИ КАК 
БЫ СФОРМИРОВАЛ «ТЕЗИС», ОБЛЕГЧИВ ГЕОРГУ МИХЕЛЬСУ ФОРМУЛИРОВКУ АНТИТЕЗИСА.

ВТОРОЙ БЛОК СТАТЕЙ, ПОСВЯЩЕННЫЙ РАННЕМУ СТАРООБРЯДЧЕСТВУ, ОТКРЫВАЕТСЯ СРАВНИТЕЛЬНО ПОЗДНЕЙ РАБОТОЙ О ЩЦЕРКОВНЫХ ЭЛИТАХ И НАРОДНЫХ ВЕРОВАНИЯХ И ПРАКТИКАХ В РОССИИ XVII В.», ОПУБЛИКОВАННОЙ В 2004 Г. ЗДЕСЬ ПОКАЗАТЕЛЬНА САМА ПО СЕБЕ ЗАМЕТКА О ТОМ, ЧТО «В ПОСЛЕДНИЕ ДЕСЯТИЛЕТИЯ СЕМНАДЦАТОГО ВЕКА ОППОЗИЦИЯ НИКОНОВСКИМ ЛИТУРГИЧЕСКИМ РЕФОРМАМ ОКАЗАЛАСЬ НЕРАЗРЫВНО СВЯЗАННОЙ С ЗАЩИТОЙ КОРПОРАТИВНЫХ ПРАВ, ЛОКАЛЬНОЙ АВТОНОМИИ ИЛИ ТРАДИЦИОННЫХ ПРАКТИК - ЗАЩИТОЙ ОТ СТАНОВЯЩИХСЯ ВСЕ БОЛЕЕ И БОЛЕЕ НАЗОЙЛИВЫМИ ВТОРЖЕНИЙ ЦЕНТРАЛЬНЫХ БЮРОКРАТИЧЕСКИХ СТРУКТУР ГОСУДАРСТВА И ЦЕРКВИ» (С. 46). ЭТА КОНСТАТАЦИЯ ПОКАЗЫВАЕТ, ЧТО КРАММИ ВНИМАТЕЛЬНО ОТНЕССЯ К ДОВОДАМ СВОИХ ОППОНЕНТОВ (НА МОЙ ВЗГЛЯД, ЭТО ЕЩЕ ОДИН АРГУМЕНТ В ПОЛЬЗУ «ХРОНОЛОГИЧЕСКОЙ» СТРУКТУРЫ СБОРНИКА). ЗАТЕМ СЛЕДУЮТ БОЛЕЕ РАННИЕ СТАТЬИ О КАПИТОНЕ ДАНИЛОВСКОМ И ЕГО «ДВИЖЕНИИ» И О ТРУДАХ СТАРООБРЯДЧЕСКОГО ПИСАТЕЛЯ АВРААМИЯ (В МИРУ ЮРОДИВОГО АФАНАСИЯ), ОПУБЛИКОВАННЫЕ СООТВЕТСТВЕННО В 1988 И 1992 ГГ. ДВИЖЕНИЕ КАПИТОНА ДАНИЛОВСКОГО ОСОБЕННО ИНТЕРЕСНО ДЛЯ ИССЛЕДОВАТЕЛЯ, ТАК КАК РЕЧЬ ИДЕТ О ГРУППЕ, ВОЗНИКШЕЙ ЕЩЕ ДО НИКОНОВСКОЙ ЛИТУРГИЧЕСКОЙ РЕФОРМЫ, НО РАЗИТЕЛЬНО НАПОМИНАЮЩЕЙ СТАРООБРЯДЧЕСТВО. КРАММИ ПРИХОДИТ ЗДЕСЬ К ВЫВОДУ О ТОМ, ЧТО КАПИТОН И ЕГО ПРИВЕРЖЕНЦЫ «МОГУТ ЗАКОННО РАССМАТРИВАТЬСЯ КАК СТАРООБРЯДЦЫ» (С. 65). ВЫВОДЫ КРАММИ ОСТОРОЖНЫ И КОРРЕКТНЫ, И ПОСКОЛЬКУ НИКАКИХ НОВЫХ ИСТОЧНИКАХ О КАПИТОНЕ И ЕГО ПОСЛЕДОВАТЛЯХ ЗА ПОСЛЕДНИЕ ДВАДЦАТЬ ЛЕТ НАЙТИ НЕ УДАЛОСЬ, ЭТА СТАТЬЯ ОСТАЕТСЯ ПОСЛЕДНИМ СЛОВОМ О ДАННОМ СЮЖЕТЕ 3 . В РАБОТЕ ОБ АВРААМИИ КРАММИ ПРИМЕНЯЕТ ПОНЯТИЯ, ПРЕДЛОЖЕННЫЕ КЛИФФОРДОМ ГИРЦЕМ - «КУЛЬТУРНАЯ СИСТЕМА» И «ФОРМИРОВАНИЕ КАНОНА». ЗАВЕРШАЕТ БЛОК СТАТЬЯ «ЧУДО МУЧЕНИЧЕСТВА», ПОСВЯЩЕННАЯ РАННЕМУ СТАРООБРЯДЧЕСТВУ, В КОТОРОЙ АВТОР ПРЕДЛАГАЕТ ТЕЗИС О ТОМ, ЧТО В ПИСАНИЯХ СТАРООБРЯДЦЕВ, СВЯЗАННЫХ С МОСКОВСКОЙ ОБЩИНОЙ (В ЖИТИИ ФЕОДОСИИ МОРОЗОВОЙ И В ТРУДАХ ТОГО ЖЕ АВРААМИЯ) АКЦЕНТ ДЕЛАЕТСЯ НА МУЧЕНИЧЕСТВЕ, В ТО ВРЕМЯ КАК В АВТОБИОГРАФИЧЕСКИХ ТЕКСТАХ ПУСТОЗЕРСКИХ УЗНИКОВ ИГРАЮТ БОЛЬШУЮ РОЛЬ ЧУДЕСА И ЧУДЕСНОЕ. ТРЕТИЙ БЛОК СБОРНИКА, ПОСВЯЩЕННЫЙ XVIII-XIX ВВ., СОСТОИТ ИЗ ПЯТИ СТАТЕЙ «СТАРООБРЯЧЕСКИЕ СООБЩЕСТВА: ИДЕАЛЫ И СТРУКТУРЫ», «ДУХОВНОСТЬ ОТЦОВ ВЫГА», «ИСТОРИЧЕСКИЕ ТРУДЫ ВЫГОВСКИХ ОТЦОВ», «КУЛЬТУРНЫЕ МИРЫ АНДРЕЯ БОРИСОВА» И «ИНТЕРПРЕТИРУЯ СУДЬБУ СТАРООБРЯДЧЕСКИХ СООБЩЕСТВ В XVIII-XIX ВВ.». НАИБОЛЕЕ ВАЖНЫМИ СРЕДИ НИХ МНЕ ПРЕДСТАВЛЯЮТСЯ СТАТЬИ О ДУХОВНОСТИ ВЫГОВСКИХ ОТЦОВ (1991) И ОБ АНДРЕЕ БОРИСОВЕ, ВОЗГЛАВЛЯВШЕМ ВЫГОВСКУЮ КИНОВИЮ В 1780-1791 ГГ. (1998). КРАММИ РАССМАТРИВАЕТ ВЫГОВСКИХ ОТЦОВОСНОВАТЕЛЕЙ В КАЧЕСТВЕ ДУХОВНЫХ НАСЛЕДНИКОВ ИОСИФЛЯН (С. 123). В КАЧЕСТВЕ ВАЖНЕЙШИХ ТЕМ ВЫГОВСКИХ ТЕКСТОВ КРАММИ ВЫДЕЛАЮТ «ВОИНСТВУЮЩИЙ АКТИВИЗМ, ДУХОВНУЮ И ФИЗИЧЕСКУЮ БОРЬБУ (warfare), ИДЕАЛ МУЧЕНИЧЕСТВА» (с. 122). ЗДЕСЬ ЖЕ ОН ЗАНИМАЕТ ПОЗИЦИЮ И ПО ВОПРОСУ О РЕГУЛЯРНО ПРИПИСЫВАЕМОЙ СТАРООБРЯДЦАМ «ПРОТЕСТАНТСКОЙ ЭТИКЕ», ОТМЕЧАЯ, ЧТО ХОЗЯЙСТВЕННАЯ ЭТИКА СТАРООБРЯДЧЕСТВА ОТТАЛКИВАЕТСЯ НЕ ОТ ТЕМЫ «ИЗБРАННОСТИ», НО «ОТ ДРЕВНЕГО ХРИСТИАНСКОГО ИДЕАЛА ДИСЦИПЛИНИРОВАНИЯ 
АППЕТИТОВ И СТРАТЕЙ, ПОДКРЕПЛЕННОГО ПРАКТИЧЕСКОЙ НЕОБХОДИМОСТЬЮ ВЫСТАИВАТЬ И ПОДДЕРЖИВАТЬ СООБЩЕСТВО ВЕРУЮЩИХ В УДАЛЕННОМ И ВРАЖДЕБНОМ ОКРУЖЕНИИ» (С. 126).

САМ ЗАГОЛОВОК СТАТЬИ ОБ АНДРЕЕ БОРИСОВЕ - Cultural worlds of Andrei BorisovОТСЫЛАЕТ К ЗАГОЛОВКУ СТАТЬИ ИГОРЯ ШЕВЧЕНКО О ПЕТРЕ МОГИЛЕ МОГИЛЕ, БОРИСОВ СТОЯЛ НА ПЕРЕСЕЧЕНИИ НЕСКОЛЬКИХ КУЛЬТУРНЫХ МИРОВ, СРЕДИ КОТОРЫХ КРАММИ ВЫДЕЛЯЕТ, ВО-ПЕРВЫХ, «ЛИТУРГИЧЕСКОЕ, ЛИТЕРАТУРНОЕ И ПОЛЕМИЧЕСКОЕ НАСЛЕДИЕ ВСЕЛЕНСКОГО ВОСТОЧНОГО ПРАВОСЛАВИЯ В ТОМ ВИДЕ, В КАКОМ ОНО БЫЛО СОХРАНЕНО И КОДИФИЦИРОВАНО В МОСКОВСКОЙ РУСИ», ВОВТОРЫХ, «НАСЛЕДИЕ САМОГО ВЫГА», И В-ТРЕТЬИХ, «ИДЕОЛОГИЧЕСКИЕ И ЛИТЕРАТУРНЫЕ ТЕЧЕНИЯ СОВРЕМЕННОГО ЕМУ МИРА» (С. 142-143). ИМЕННО ПРОСЛЕЖИВАЯ ВЗАИМООТНОШЕНИЯ БОРИСОВА С ЭТИМИ ТЕЧЕНИЯМИ, КРАММИ СОЗДАЕТ ЭТЮД, ОТВЕЧАЮЩИЙ ТЕМЕ КНИГИ - СТАРООБРЯДЧЕСТВУ В МЕНЯЮЩЕМСЯ МИРЕ. МОЖНО ТОЛЬКО СОГЛАСИТЬСЯ С ВЫВОДОМ КРАММИ О ТОМ, ЧТО БОРИСОВ ИСПЫТАЛ ВЛИЯНИЕ МАСОНСКИХ ТРАКТАТОВ О САМОСОВЕРШЕНСТВОВАНИИ. НЕ СЛУЧАЕН И СОЧУВСТВЕННЫЙ ИНТЕРЕС БОРИСОВА К ТРУДАМ ВОЛЬТЕРА И РУССО (КОТОРЫЕ ОН, ЕСТЕСТВЕННО, ЧИТАЛ В РУССКИХ ПЕРЕВОДАХ). БЕЗ Traité sur la tolérance (1763) И ВЫСТУПЛЕНИЙ ФИЛОСОФА В ЗАЩИТУ СЕМЬИ КАЛА НЕ БЫЛО БЫ НИ ВЕРОТЕРПИМОЙ ПОЛИТИКИ ЕКАТЕРИНЫ II В ЦЕЛОМ, НИ ОТМЕНЫ ДВОЙНОГО ПОДУШНОГО ОКЛАДА НА СТАРООБРЯДЦЕВ В ЧАСТНОСТИ. С ЛЕГКОЙ ИРОНИЕЙ ПЕРЕДАЕТ КРАММИ ВОСПОМИНАНИЯ ЯКОБИНЦА ЖИЛЬБЕРА РОММА, ВОЛЕЮ СУДЬБЫ ЗАНЕСЕННОГО В КАРЕЛЬСКИЕ ЛЕСА, О ВСТРЕЧАХ С АНДРЕЕМ БОРИСОВЫМ. РОММУ, ЧЕЛОВЕКУ НОВОГО ПОКОЛЕНИЯ, УЖЕ НЕСВОЙСТВЕННА БЕЗУСЛОВНАЯ ПРОСВЕТИТЕЛЬСКАЯ СОЛИДАРНОСТЬ СО ВСЯКИМ ВЕРОУЧЕНИЕМ, ПРЕСЛЕДУЕМЫМ СВЫШЕ, И ОН СТАРАЕТСЯ ВТИСНУТЬ СВОИ ВПЕЧАТЛЕНИЯ ОТ ВСТРЕЧИ СО СТАРООБРЯДЧЕСКИМ НАСТАВНИКОМ В ГОТОВЫЙ АНТИКЛЕРИКАЛЬНЫЙ ДИСКУРС.

ОШИБКИ И ФАКТИЧЕСКИЕ НЕТОЧНОСТИ МОГУТ БЫТЬ НАЙДЕНЫ В ЛЮБОМ ИССЛЕДОВАНИИ, НО В РЕЦЕНЗИРУЕМОЙ КНИГЕ ИХ СОВСЕМ НЕМНОГО. КРАММИ НАЗЫВАЕТ СПИРИДОНА ПОТЕМКИНА АРХИМАНДРИТОМ ПОКРОВСКОГО МОНАСТЫРЯ НА УБОГИХ МОНАСТЫРЯХ (С. 71) (СПРАВЕДЛИВОСТИ РАДИ, ОТМЕТИМ, ЧТО ЭТО УКАЗАНИЕ ПОПАЛО ВО ВСЕ СПРАВОЧНИКИ, ВКЛЮЧАЯ СПИСКИ ИЕРАРХОВ П.М. СТРОЕВА). НА САМОМ ДЕЛЕ, НИКАКОГО АРХИМАНДРИТА В ОПИСЫВАЕМОЕ ВРЕМЯ В МОНАСТЫРЕ НЕ БЫЛО, А БЫЛ СТРОИТЕЛЬ. ВНИМАТЕЛЬНОЕ ЧТЕНИЕ ЧЕЛОБИТНОЙ РЯЗАНСКОГО АРХИЕПИСКОПА ИЛАРИОНА (НЕ РАНЕЕ 18 НОЯБРЯ 1665 Г.), ОПУБЛИКОВАННОЙ В.С. РУМЯНЦЕВОЙ, УКАЗЫВАЕТ, ЧТО СТРОИТЕЛЮ ПОКРОВСКОГО МОНАСТЫРЯ КИРИЛЛУ СОБИРАЛИСЬ ДАТЬ ПОРУЧЕНИЕ, «ЧТОБ ОН СПИРИДОНУ ПОТЕМКИНУ ВОЛИ НЕ ДАВАЛ ПИСАТЬ И ЛЮДЕМ К НЕМУ ПРИХОДИТЬ». СЛЕДОВАТЕЛЬНО, ПО КРАЙНЕЙ МЕРЕ В ОПИСАННОЕ ВРЕМЯ СПИРИДОН ПОТЕМКИН БЫЛ НЕ ГЛАВОЙ ОБИТЕЛИ, А СКОРЕЕ ПОДНАДЗОРНЫМ ИНОКОМ. «СВЯЩЕННИК ДОРОФЕЙ», ПРИЧАЩАЮЩИЙ МОРОЗОВУ, УРУСОВУ И ДАНИЛОВУ (С. 94), ЭТО, СКОРЕЕ ВСЕГО, ВСЕ-ТАКИ ИГУМЕН ДОСИФЕЙ ЧИРСКИЙ

8 Я НЕ СОГЛАСЕН С МНЕНИЕМ КРАММИ, ЧТО АРХИВ ПРИКАЗА ТАЙНЫХ ДЕЛ ЯВЛЯЕТСЯ «ЧАСТНЫМ АРХИВОМ ЦАРЯ АЛЕКСЕЯ МИХАЙЛОВИЧА», СОДЕРЖИТ ЛИШЬ «ДОКУМЕНТАЦИЮ О ДВОРЦОВЫХ ИМЕНИЯХ» И НЕ МОЖЕТ СЛУЖИТЬ ОСНОВОЙ ДЛЯ ГЕНЕРАЛЬНЫХ ВЫВОДОВ (С. 66). НА МОЙ ВЗГЛЯД, ПРИКАЗ ТАЙНЫХ ДЕЛ ИМЕЛ ОПРЕДЕЛЕННУЮ КОМПЕТЕНЦИЮ В СФЕРЕ ПРЕСЛЕДОВАНИЯ РЕЛИГИОЗНОГО РАЗНОМЫСЛИЯ, КОТОРАЯ ПОСЛЕ РОСПУСКА ПРИКАЗА БЫЛА УНАСЛЕДОВАНА ДРУГИМИ 
ВЕДОМСТВАМИ. НЕ ВЕРЮ Я И В ИСТОРИЧНОСТЬ ТАЙНОГО СТАРООБРЯДЧЕСКОГО СОБОРА 1654 Г. (С. 74). ЭТОТ СОБОР, ПЕРВЫЙ РАЗ ПОЯВЛЯЮЩИЙСЯ В СРАВНИТЕЛЬНО ПОЗДНИХ ИСТОЧНИКАХ (НАПРИМЕР, В ЖИТИИ КОРНИЛИЯ ВЫГОВСКОГО), КАЖЕТСЯ НИКОГДА НЕ СОБИРАЛСЯ. ВПРОЧЕМ, РЕЧЬ ЗДЕСЬ СКОРЕЕ ИДЕТ О ЧАСТНОСТЯХ. НОВАЯ КНИГА РОБЕРТА КРАММИ ЯВЛЯЕТСЯ ВПЕЧАТЛЯЮЩИМ ОТЧЕТОМ О БОЛЕЕ ЧЕМ ТРИДЦАТИЛЕТНЕЙ РАБОТЕ НАД ТЕМОЙ, РАВНЫМ ОБРАЗОМ ПРЕДНАЗНАЧЕННЫМ И ДЛЯ СПЕЦИАЛИСТОВ, И ДЛЯ ТЕХ, КТО ВПЕРВЫЕ ОБРАЩАЕТСЯ К ТЕМЕ.

\section{NOTES}

1. Robert O.Crummey, The Old Believers and the World of Antichrist: The Vyg Community and the Russian State, 1694-1855, Madison - Milwaukee - London, 1970; Idem, Aristocrats and Servitors: The Boyar Elite in Russia, 1613-1689, Princeton, 1983; Idem, The Formation of Muscovy, 1300-1613, London, 1987.

2. G. Scheidegger Endzeit: Russland am Ende des 17. Jahrhunderts, Bern, 1999. ПУБликАциям ИСТОРИКОВ, ПИШУЩИХ ПО-НЕМЕЦКИ, В ЭТОМ ОБЗОРЕ ВООБЩЕ НЕ ПОВЕЗЛО. ЗДЕСЬ НЕТ ДВУХ МОНОГРАФИЙ ПЕТЕРА ХАУПТМАННА, АВТОРА ВИРТУОЗНОГО ПЕРЕВОДА "ЖИТИЯ" ПРОТОПОПА АВВАКумА, Р. Hauptmann, Altrussischer Glaube Der Kampf des Protopopen Avvakum gegen die Kirchenreformen des 17. Jahrhunderts, Göttingen, 1963; Idem, Russlands Altgläubige, Göttingen, 2005.

3. ИСКЛЮЧЕНИЕ МОГ БЫ СОСТАВИТЬ НАЙДЕННЫЙ А.В. БОРОДКИНЫМ ПОЛЕМИЧЕСКИЙ ТРАКТАТ ПРОТИВ «КАПИТОНОВ», ОТНОСЯЩИЙСЯ К 1680-1690-М ГГ., НО, К СОЖАЛЕНИЮ, АВТОР НАСТОЛЬКО НЕУДАЧНО ВВЕЛ ИСТОЧНИК В НАУЧНЫЙ ОБОРОТ, ЧТО НЕ СОВСЕМ ПОНЯТНЫМ ОСТАЕТСЯ ТО, ПРОТИВ КОГО ОН БЫЛ НАПРАВЛЕН - СОБСТВЕННО ПРОТИВ ПОСЛЕДОВАТЕЛЕЙ КАПИТОНА ИЛИ ПРОТИВ СТАРООБРЯДЦЕВ, КОТОРЫХ ИНОГДА НАЗЫВАЛИ «КАПИТОНАМИ» (А.В. БОРОДКИН, «ИСТОРИЯ ОДНОЙ ФАЛЬСИФИКАЦИИ: РАНЕЕ НЕ ИЗВЕСТНЫЙ ПАМЯТНИК ИНФОРМАЦИОННОЙ ВОЙНЫ ПРАВИТЕЛЬСТВА ПРОТИВ СТАРООБРЯДЦЕВ ВО ВТОРОЙ ПОЛОВИНЕ XVII В. [A.V. Borodkin, Histoire d'une falsification : Une source inconnue sur la guerre d'information du gouvernement contre les vieux-croyants dans la seconde moitié du XVII ${ }^{e}$ siècle]», СТАРООБРЯДЧЕСТВО: ИСТОРИЯ, КУЛЬТУРА, СОВРЕМЕННОСТЬ. МАТЕРИАЛЫ [Les vieux-croyants: Histoire, culture, présent. Matériaux]. M., 2000, C. 285-289).

4. Ihor Shevchenko, "The Many Worlds of Peter Mohyla," Harvard Ukrainian Studies, 8 (1-2), June 1984, p. 9-44.

5. ПОВЕСТЬ О БОЯРЫНЕ МОРОЗОВоЙ [Récit sur la bojarynja Morozova]. ПОДГОТОВКА ТЕКСТА И ИССЛЕДОВАНИЕ А.И. МАЗУНИНА, Л., 1979, С. 132, 153. 